\title{
Evisceración vaginal tras tratamiento con radioterapia e histerectomía abdominal
}

\author{
Isabel González B. ${ }^{1}$, María Ángeles Aragón S. ${ }^{1}$, Teresa Arribas M. ${ }^{1}$, Lorena Guardia \\ $D .^{1}$, Beatriz Rojas $P .^{1}$ \\ ${ }^{1}$ Servicio de Obstetricia y Ginecología. Hospital de Barbastro, Huesca. España.
}

\section{RESUMEN}

La evisceración vaginal es una complicación muy rara. Es más frecuente en mujeres posmenopáusicas y con antecedente de cirugía vaginal, fundamentalmente histerectomía. También puede darse en mujeres premenopáusicas, vinculándose en estos casos a traumatismos, iatrogenia o introducción de cuerpos extraños. El íleon distal es el órgano más frecuentemente eviscerado, aunque el prolapso de epiplón, trompas de Falopio y apéndice también se han descrito. Presentamos el caso de una mujer de 43 años con evisceración transvaginal de epiplón a los seis meses de realizarse una histerectomía abdominal por recidiva de un cáncer escamoso de cérvix.

\section{PALABRAS CLAVE: Evisceración vaginal, histerectomía, dehiscencia, cúpula vaginal}

\section{SUMMARY}

Vaginal evisceration is a very rare complication. It is more often in postmenopausal women with a history of vaginal surgery, mainly hysterectomy. It can also occur in premenopausal women, linking these cases to trauma, iatrogenic or foreign bodies. The distal ileum is most often gutted organ prolapse, although omentum, fallopian tubes and appendix are also described. We report a 43-year old transvaginal omental evisceration after six months of an abdominal hysterectomy for recurrent squamous cervical cancer.

\section{KEY WORDS: Vaginal evisceration, hysterectomy, dehiscence, vaginal cuff}

\section{INTRODUCCIÓN}

Se define evisceración como la salida de vísceras abdominales, quedando expuestas, a través de un orificio no natural (1). Es una complicación poco frecuente. Su incidencia, oscila entre 0,03 y $4,1 \%$ (2). Partsinevelos y cols en 2009 , publicaron una revisión de 51 casos donde el mayor porcentaje de casos se produjo tras histerectomía abdominal (47\%), luego de histerectomía vaginal $(29,4 \%)$ y por último después de abordaje laparoscópico (23,6\%) (3). Un estudio en 2012 señalaba una incidencia mayor de dehiscencia de cúpula vaginal después de histerectomía laparoscópica $(1,14 \%)$ que tras histerectomía abdominal $(0,10 \%)$ y vaginal $(0,14 \%)(4)$.

Analizando los diferentes abordajes laparoscópicos de la histerectomía, la incidencia de evisceración es mayor en la histerectomía total laparoscópica que en la histerectomía vaginal asistida por laparoscopia, y la sutura intracorpórea de la cúpula vaginal en la histerectomía laparoscópica obtiene mejores resultados que la sutura vía vaginal (5). 
El objetivo de esta comunicación es presentar un caso de evisceración vaginal tras tratamiento con radioterapia e histerectomía abdominal.

\section{Caso clínico}

Paciente de 43 años que presentaba como antecedentes obstétricos dos partos eutócicos y como antecedentes personales, obesidad y cáncer de cérvix escamoso infiltrante diagnosticado en 2009. Fue intervenida quirúrgicamente de esta patología, realizándose biopsia selectiva de ganglio centinela con resultado positivo, por lo que se procedió a linfadenectomía paraaórtica y pélvica bilateral con pexia ovárica bilateral y conservación de útero in situ por estadío IIB. El examen anatomopatológico concluyó la afectación de dos ganglios iliacos izquierdos. Se completó con tratamiento adyuvante con quimioterapia y radioterapia externa y braquiterapia concomitantes, presentando un cuadro de radiodermitis infecciosa tardía que precisó ingreso y desarrollo de linfedema crónico de extremidad inferior derecha.

Se realizó seguimiento, siendo todos los controles posteriores negativos hasta cinco años después, donde se obtiene citología con resultado de lesión escamosa intraepitelial de alto grado (HSIL) y virus del papiloma humano positivo a virus de alto riesgo no 16 ni 18 . Se decide nuevo control en tres meses, con citología con resultado de lesión escamosa intraepitelial de alto grado (HSIL). Toma de biopsias múltiples de ectocérvix con diagnóstico de lesión escamosa intraepitelial de alto grado (CIN3) y legrado endocervical con fragmentos de lesión escamosa intraepitelial de bajo grado (CIN1).

Se solicitan como pruebas complementarias, radiografía de tórax, resonancia magnética nuclear abdomino-pélvica, mamografía, análisis con marcadores tumorales y tomografía por emisión de positrones (PET-TAC), siendo negativas todas ellas.

Ante los resultados, se decide realizar rescate quirúrgico con histerectomía total simple vía abdominal. El estudio anatomopatológico informa de lesión escamosa intraepitelial de alto grado (CIN2CIN3) a nivel de toda la circunferencia ectoendocervical sin afectación de extremos quirúrgicos. El postoperatorio transcurre con buena evolución recibiendo el alta al quinto día. Al mes de la intervención presenta un cuadro de trombosis venosa profunda de la extremidad inferior derecha que precisa ingreso resolviéndose satisfactoriamente. El último control citológico de cúpula vaginal a los cinco meses poscirugía informa de células escamosas atípicas de significado incierto (ASC-US).

A los seis meses, acude al servicio de Urgencias de Obstetricia y Ginecología por leucorrea sanguinolenta de tres días de evolución con ligero prurito genital y sensación de bulto en genitales. Refiere que en los días previos durante una relación sexual tuvo dolor intenso y brusco con manchado genital posterior. A la exploración se aprecian genitales externos normales, vagina con efectos de radioterapia, leucorrea inespecífica con restos hemáticos escasos y lesión exofítica lisa, indurada y sangrante al roce, de aproximadamente $5 \mathrm{~cm}$ que asoma por introito (Figura 1). Al tacto vaginal, ocupa toda la vagina. Se piensa en diagnóstico diferencial de granuloma de cicatriz o recidiva tumoral por lo que se toma citología con resultado de atrofia y biopsia con resultado de tejido reactivo y necrosado (Figura 2). Ante este resultado se cita a la paciente para informarle y se decide programar exploración bajo anestesia en quirófano con ampliación de muestra de biopsia en dos semanas. En quirófano con la paciente anestesiada se objetiva una lesión exofítica de menor tamaño $(2 \times 1 \mathrm{~cm})$, de aspecto graso, polipoide, friable y sangrante al roce, que parece corresponder a apéndice epiploico. Al traccionar con pinza de Foerster se produce salida de epiplón y asas intestinales de delgado, ampliándose espontáneamente lo que era un defecto en cúpula vaginal de $1 \mathrm{~cm}$ hasta $3 \mathrm{~cm}$. Se comprobó la viabilidad de las asas intestinales, se realizó lavado de las mismas con suero fisiológico y se procedió a su introducción a través de la cúpula vaginal abierta.

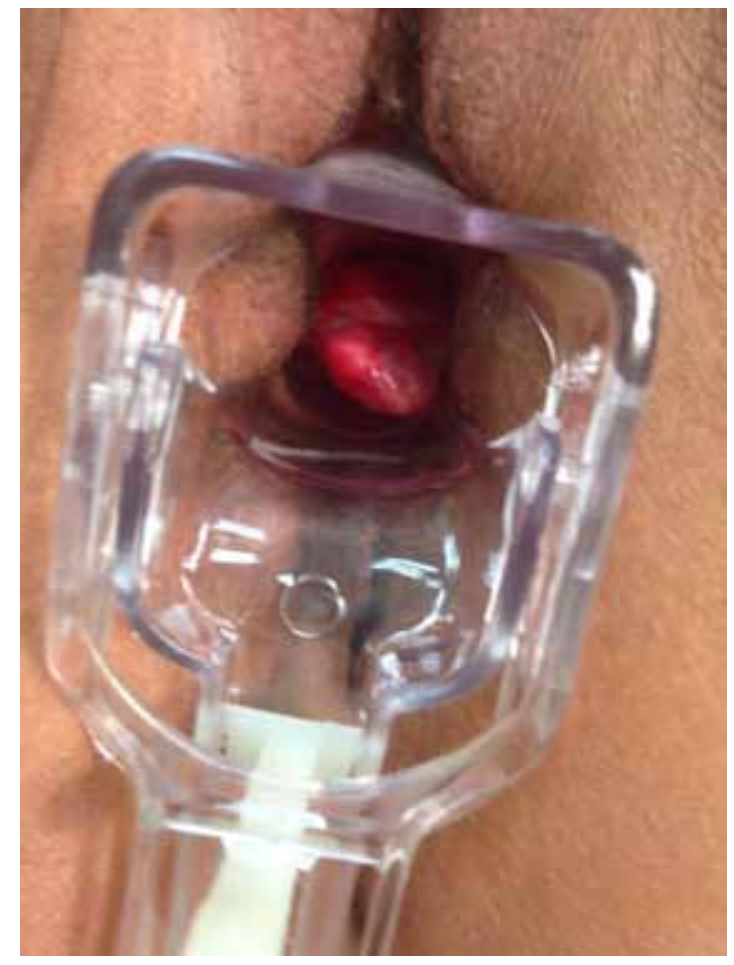

Figura 1. Lesión exofítica lisa, indurada y sangrante al roce de aproximadamente $5 \mathrm{~cm}$ que ocupa al tacto toda la vagina y asoma por introito. 


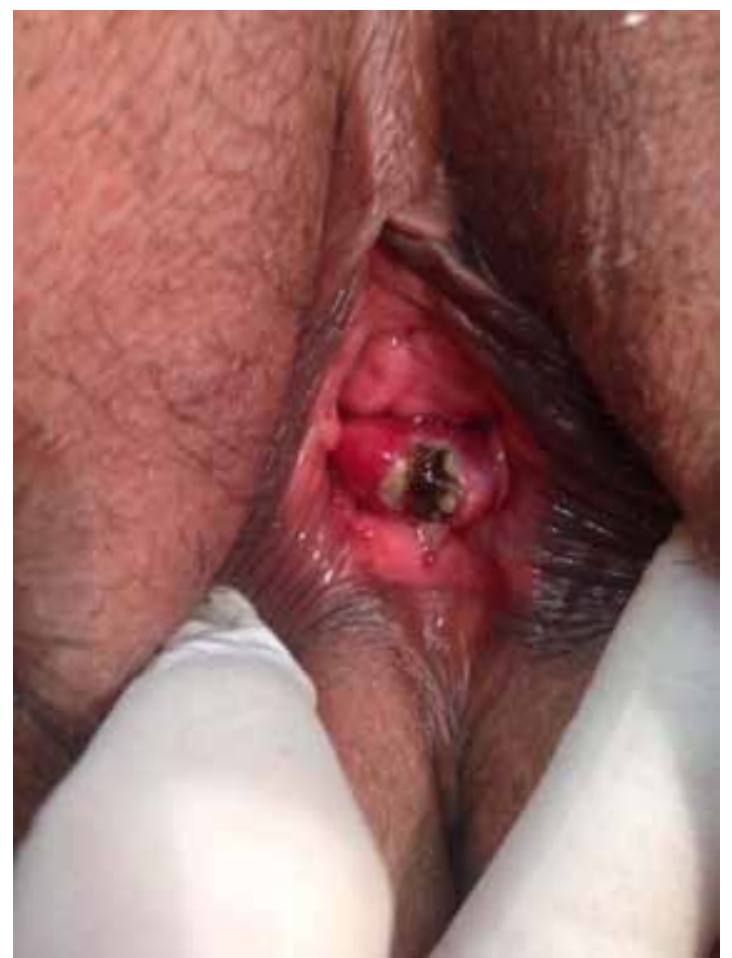

Figura 2. Imagen de la lesión tras realizar biopsia con pinza sacabocados y coagulación con nitrato de plata.

Posteriormente, ante la facilidad en la reducción y la viabilidad intestinal se procedió a la sutura del defecto vía vaginal con sutura absorbible sintética de ácido poliglicólico (Vycrilß), quedando la cúpula vaginal bien suspendida. La evolución posoperatoria fue favorable, recibiendo la paciente el alta a las 48 horas de la intervención. Al mes, fue revisada en consultas externas constatándose la no existencia de dehiscencia de la cicatriz (Figura 3). Se prescriben óvulos de estriol 0,5 mg para mejorar el trofismo de la mucosa vaginal.

\section{DISCUSIÓN}

Lo insólito del caso que presentamos es el buen estado de la paciente, al tratarse de una evisceración de epiplón y no afectar a asas intestinales, viniendo de forma programada a exploración bajo anestesia cuando el defecto de la cúpula vaginal llevaba al menos dos semanas. El íleon terminal es la víscera implicada más frecuentemente, pero también se han publicado casos más raros de salida de epiplón, trompas de Falopio, apéndice y un único caso de quiste ovárico (6).

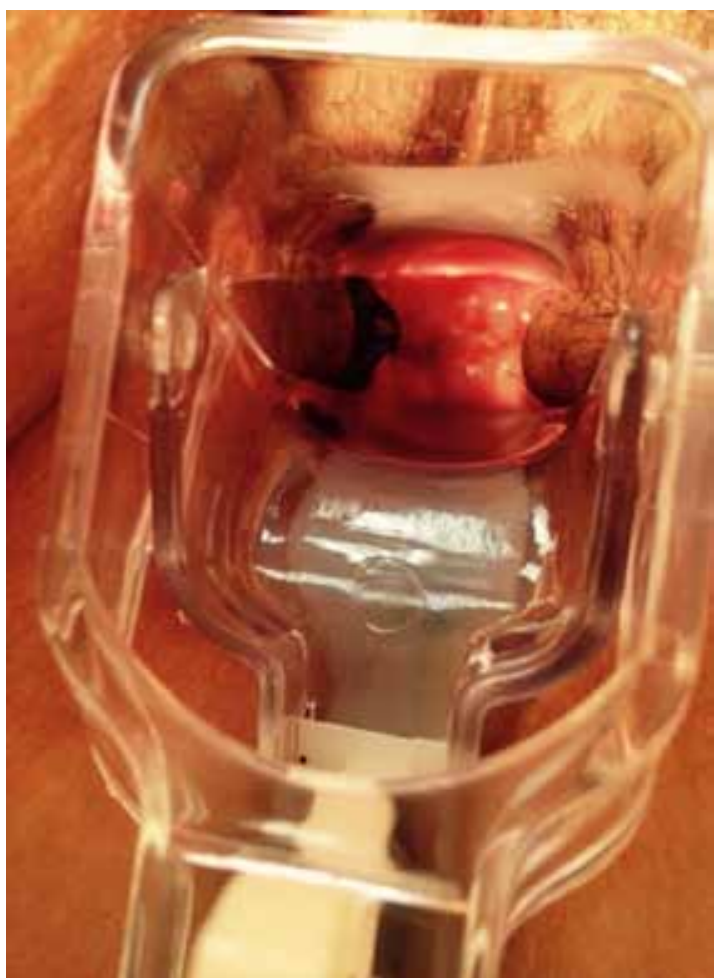

Figura 3. Aspecto de la cúpula vaginal sin dehiscencia y bien suspendida en la revisión al mes de la reducción de la evisceración.

La evisceración transvaginal se asocia a diversos factores. El principal es el antecedente quirúrgico, principalmente la histerectomía, bien por vía vaginal, abdominal o laparoscópica. Otros factores de riesgo son el estado hipoestrogénico que produce atrofia de la cúpula vaginal, la mala técnica quirúrgica, infección o hematoma posquirúrgicos, el coito antes de la cicatrización completa, maniobras de Valsalva, la edad, el tabaco, el tratamiento crónico con corticosteroides y la radioterapia (7).

En nuestro caso la paciente recibió tratamiento con radioterapia externa y braquiterapia adyuvantes por un carcinoma de cérvix. En 2008, Sánchez y cols publicaron el caso de una evisceración secundaria a cirugía y radioterapia por un cáncer cervicouterino (8). Tras la radioterapia el tejido queda con una vascularización reducida lo que da lugar a un tejido hipóxico e hipocelular y en definitiva, débil, lo que puede producir una rotura tisular de forma espontánea o tras un traumatismo directo (9). En mujeres premenopáusicas se asocia a traumatismos durante el coito, violación, introducción de cuerpos extraños o iatrogenia (10). El intervalo de 
tiempo entre el acto quirúrgico y la complicación varía según la literatura publicada desde un día hasta 25 años (11). En nuestro caso habían transcurrido seis meses.

El cuadro clínico se caracteriza por dolor a nivel vaginal o pélvico, hemorragia vaginal o aparición de una masa que protruye a través de la vagina. Nuestra paciente presentaba leucorrea sanguinolenta con ligero prurito genital y sensación de bulto en genitales, sin dolor pélvico. En pacientes que presentan una evisceración franca de asas intestinales, se trata de una emergencia quirúrgica por el alto riesgo de necrosis y/o perforación intestinal y de sepsis (12). En el caso que presentamos la paciente se encontraba con buen estado general, estable hemodinámicamente, sin dolor pélvico y ante la sospecha de posible recidiva tumoral no se realizó cirugía de urgencia.

La vía de abordaje para la reparación del defecto vaginal depende del grado de herniación, de la estabilidad hemodinámica de la paciente y de la viabilidad intestinal. La vía laparotómica clásica permite inspeccionar totalmente el intestino y el mesenterio con un buen campo quirúrgico. Sin embargo, en casos como el que presentamos en que los signos y síntomas abdominales sean mínimos o ausentes, la evisceración sea fácilmente reducible y las asas intestinales sean claramente viables se puede optar por la vía vaginal, evitando así la morbilidad de la laparotomía (13).

En 2012, se publicó una revisión de 73 casos de reparación de dehiscencia vaginal con evisceración intestinal. Los resultados mostraron un $51 \%$ de casos con reparación vía vaginal, $32 \%$ vía abdominal, $2 \%$ vía laparoscópica, 10\% combinada (abdominal y vaginal o laparoscópica y vaginal) y $5 \%$ cicatrización por segunda intención (14).

Acerca del momento adecuado para realizar la corrección de la disfunción del suelo pélvico existe controversia entre los autores. Algunos defienden la reparación en el mismo acto quirúrgico y otros creen que es mejor la opción de corregirlo en un segundo tiempo, puesto que disminuye el riesgo de infección, aunque aumenta el riesgo quirúrgico y anestésico por una segunda intervención (15). En muchos casos suele ser suficiente el cierre simple de la cúpula vaginal, aunque se puede colocar una malla de politetrafluoroetileno o polipropileno, ambos materiales irreabsorbibles, para reforzar la sutura. En pacientes oncológicas, como es nuestro caso, no se recomienda su colocación debido a la deficiente calidad de los tejidos (16). Para reforzar la cúpula vaginal se pueden utilizar los ligamentos úterosacros o cardinales, realizar una colposacropexia, la técnica de Moschcowitz o la culdoplastía modificada de McCall (17).

\section{CONCLUSIÓN}

La evisceración transvaginal es una complicación poco frecuente de la histerectomía pero que requiere un diagnóstico y tratamiento urgente para evitar la morbimortalidad asociada. La realización de cirugías con una técnica quirúrgica depurada y el tratamiento correcto de las disfunciones del suelo pélvico pueden evitar su aparición.

\section{REFERENCIAS}

1. Martí Carvajal P, Pineda E, Martí C. Evisceración vaginal posthisterectomía vaginal. A propósito de un caso. Academia Biológica Digital 2010; Vol 0: No 44. Disponible en: http://vitae.ucv.ve/?module=articulo\&r $v=97 \& n=4269$.

2. Nguyen ML, Kapoor M, Pradhan TS, Pua TL, Tedjarati SS. Two cases of post-coital vaginal cuff dehiscence with small bowel evisceration after robotic-assisted laparoscopic hysterectomy. Int J Surg Case Rep 2013;4(7):603-5.

3. Partsinavelos G, Rodolakis A, Athanasiou S, Antsaklis A. Vaginal evisceration after hysterectomy: a rare condition a gynecologist should be familiar with. Arch Gynecol Obstet 2009;279:267-70.

4. Liao $\mathrm{CH}$, Sung SY, Lin HL. Peritonitis caused by vaginal evisceration following laparoscopy-assisted vaginal hysterectomy. Taiwan J Obstet Gynecol 2013;52(2):285-6.

5. Myung JK, Seongmin K, Hyo SB, Jae KL, Nak WL, Jae YS. Evaluation of risk factors of vaginal cuff dehiscence after hysterectomy. Obstet Gynecol Sci 2014;57(2):136-43.

6. Nguyen ML, Anyikam AL, Paolucci M. Vaginal cuff dehiscence with adnexal mass evisceration after abdominal hysterectomy. Int J Surg Case Rep 2013;4(5):518-20.

7. Ramírez PT, Klemer DP. Transvaginal evisceration after hysterectomy: a literature review. Obstet Gynecol Surv 2002;57(7):462-7.

8. Sánchez HJ, Naranjo TA, Ciria BR, Gallardo BJ, Rufián PS. Evisceración vaginal. Cir Esp 2008;84:10111.

9. Kang WD, Kim SM, Choi HS. Vaginal evisceration after radical hysterectomy and adjuvant radiation. $\mathrm{J}$ Gynecol Oncol 2009;20(1):63-4.

10. Quiroz Guadarrama CD, Martínez Ordaz JL, Rojano Rodríguez ME, Beristain Hernández JL, Moreno Portillo M. Evisceración vaginal. Informe de un caso y revisión de la bibliografía. Ginecol Obstet Mex 2013;81(6):349-52.

11. Hur HC, Guido RS, Mansuria SM, Haacker MR, Sanfilippo JS, Lee TT. Incidence and patient characteristics of vaginal cuff dehiscence after different modes of hysterectomies. J Minim Invasive Gynecol 2007;14(3):311-7. 
12. Cabezas Palacios MN, Guadix Martín P, Gómez Rosado JC, Jiménez Caraballo AJ. Evisceración transvaginal posthisterectomía radical y radioterapia adyuvante. Prog Obstet 2012;55(1):20-3.

13. Ricotta A, Sofia M, Latteri S, Lomeo E, Rossello D, La Greca G. Transvaginal evisceration 20 years after hysterectomy. Case report and literature review. Ann Ital Chir 2014; 20:85.(ePub). pii: S2239253X14022178.

14. Cronin B, Sung VW, Matteson KA. Vaginal cuff dehiscence: risk factors and management. Am J Obstet Gynecol 2012;206(4):284-8.
15. Dabrowiecki S. Abdominal wall closure techniquesthe results of the Polish surgeons' survey. Pol Merkur Lekarski 2005;19(113):646-50.

16. Narducci F, Sonoda Y, Lambaudie E, Leblanc E, Querleu D. Vaginal evisceration after hysterectomy: the repair by a laparoscopic and vaginal approach with omental flap. Gynecol Oncol 2003; 89(3):549-51.

17. Mastrolia SA, Di Naro E, Schonauer LM, Loverro MT, Indellicati $B$, Barnaba $M$, et al. Vaginal treatment of vaginal cuff dehiscence with visceral loop prolapse: a new challenge in reparative vaginal surgery? Case Rep Obstet Gynecol 2014;2014:257398. 